\title{
A comprehensive platform for highly multiplexed mammalian functional genetic screens
}

\author{
Troy Ketela ${ }^{1 \dagger}$, Lawrence E Heisler ${ }^{1 \dagger}$, Kevin R Brown ${ }^{1}$, Ron Ammar $^{1,2}$, Dahlia Kasimer ${ }^{1}$, Anuradha Surendra ${ }^{1}$, \\ Elke Ericson ${ }^{1}$, Kim Blakely ${ }^{1,2}$, Dina Karamboulas ${ }^{1}$, Andrew M Smith ${ }^{1,2}$, Tanja Durbic ${ }^{1}$, Anthony Arnoldo', \\ Kahlin Cheung-Ong ${ }^{1}$, Judice LY Koh', Shuba Gopal ${ }^{3}$, Glenn S Cowley ${ }^{3}$, Xiaoping Yang ${ }^{3}$, Jennifer K Grenier ${ }^{3}$, \\ Guri Giaever ${ }^{1,2,4}$, David E Root ${ }^{3 *}$, Jason Moffat ${ }^{1,2^{*}}$ and Corey Nislow ${ }^{1,2^{*}}$
}

\begin{abstract}
Background: Genome-wide screening in human and mouse cells using RNA interference and open reading frame over-expression libraries is rapidly becoming a viable experimental approach for many research labs. There are a variety of gene expression modulation libraries commercially available, however, detailed and validated protocols as well as the reagents necessary for deconvolving genome-scale gene screens using these libraries are lacking. As a solution, we designed a comprehensive platform for highly multiplexed functional genetic screens in human, mouse and yeast cells using popular, commercially available gene modulation libraries. The Gene Modulation Array Platform (GMAP) is a single microarray-based detection solution for deconvolution of loss and gain-of-function pooled screens.

Results: Experiments with specially constructed lentiviral-based plasmid pools containing 78,000 shRNAs demonstrated that the GMAP is capable of deconvolving genome-wide shRNA "dropout" screens. Further experiments with a larger, $\sim 90,000$ shRNA pool demonstrate that equivalent results are obtained from plasmid pools and from genomic DNA derived from lentivirus infected cells. Parallel testing of large shRNA pools using GMAP and next-generation sequencing methods revealed that the two methods provide valid and complementary approaches to deconvolution of genome-wide shRNA screens. Additional experiments demonstrated that GMAP is equivalent to similar microarray-based products when used for deconvolution of open reading frame overexpression screens.
\end{abstract}

Conclusion: Herein, we demonstrate four major applications for the GMAP resource, including deconvolution of pooled RNAi screens in cells with at least 90,000 distinct shRNAs. We also provide detailed methodologies for pooled shRNA screen readout using GMAP and compare next-generation sequencing to GMAP (i.e. microarray) based deconvolution methods.

\section{Background}

Beginning in the late 1990's, construction of a systematic, barcoded gene deletion strain collection in S. cerevisiae set the stage for high throughput functional screening in eukaryotes [1,2]. Researchers using that resource developed powerful functional genomics

\footnotetext{
* Correspondence: droot@broadinstitute.org; j.moffat@utoronto.ca; corey. nislow@utoronto.ca

† Contributed equally

${ }^{1}$ Donnelly Centre and Banting \& Best Department of Medical Research, University of Toronto, Toronto, Canada

${ }^{3}$ Broad Institute, Cambridge, USA Full list of author information is available at the end of the article
}

screening approaches, and significantly, introduced the concept of performing complex, pooled population screens to simultaneously interrogate an organism's entire genome to identify drug targets, synthetic genetic interactions and other phenomena [1,3-7]. Similarly, the contemporary development of large libraries of short hairpin RNAs (shRNAs) and open reading frame (ORF) collections has significantly expanded the research toolkit available for performing mammalian functional genetics in a comprehensive manner [8-11]. The development of such tools, combined with the years of lessons from yeast-based screens suggested that similar genome-wide screens using a 'barcoded pool' screening
C Biomed Central 
strategy should be possible in a mammalian context $[12,13]$. Indeed, several groups have demonstrated the impressive ability of pooled RNAi dropout screens to identify genes essential to cancer cell proliferation [14-18]. Based on these early reports, pooled RNAi screens hold great promise for identifying genes important for growth, metabolism, differentiation, DNA damage response, sensitivity/resistance to therapeutics and many other processes in both normal and diseased cells.

Linking phenotypic changes to gene dosage in a heterogeneous population of cells requires a stably inherited molecular tag, such as a DNA barcode, that uniquely associates the phenotype with the perturbagen. A decade of work with yeast and other microbes has shown that DNA barcodes are sensitive and quantitative genetic markers that permit cell-based screening in high complexity pooled formats, with subsequent deconvolution by amplification of barcodes followed by microarray hybridization or high throughput DNA sequencing [19-21]. In contrast to the yeast knock-out strains, for pooled shRNAs and ORFs the growth perturbing agents themselves can serve as specific barcodes because they consist of uniquely identifiable DNA sequences. This approach has been demonstrated in several recent publications using different shRNA libraries [14-17]. For each publication, a different custom array was built and quality controlled, but information on the optimization and validation was limited, and the microarrays were not made commercially available. Consequently, researchers interested in utilizing this technology would need to perform resource intensive optimization and quality assurance on a new custom microarray for each shRNA library - an approach that is cost-inefficient, and does not promote standardized datasets amenable to high level informatics analysis. In order to facilitate loss-offunction and gain-of-function pooled screens using commercially available gene modulation libraries, we designed and validated a single microarray detection platform for deconvolution of loss- and gain-of-function pooled screens in human, mouse and yeast cells.

One can now easily procure custom microarrays designed to detect novel barcoded libraries. In order to evaluate and maximize the effectiveness of such barcoded platforms, extensive testing and optimization is required. For gene expression studies, extensive development work has already been done for a number of detection platforms and, as a result, they have become straightforward and routine to use. For new custom barcoded systems, similarly rigorous testing, optimization of protocols, and development of quality control standards must be done. This manuscript describes a detailed evaluation and analysis of the performance of the Gene Modulation Array Platform (GMAP) microarray, and carefully optimized protocols for every step of the process from sample preparation to data analysis. In essence, we provide a guide to use the GMAP chip as well as highlight the design principles and quality control measures that may be useful for other customized microarray platforms. In addition, we have also developed a software tool (using open source scripts) for use in extracting and analysing the data from the GMAP.

\section{Results and Discussion}

We designed the multipurpose, cost-effective GMAP to enable researchers to inexpensively and comprehensively collect data from genome-scale pooled gene-dosage modulation screens performed in human, mouse, and yeast cells using commercially available gene modulation libraries on a standard platform. Specifically, the GMAP enables readout of clone enrichments and depletions from pooled screens using the RNAi consortium (TRC) human and mouse libraries $[11,14,22]$, human ORF expression pools $[23,24]$, and pooled screens using gene deletion-associated barcodes or ORFs from budding yeast $[1,25]$.

The detection features on GMAP are summarized in Table 1. The GMAP features encompass the entire TRC1 shRNA human and mouse collections and a portion of the greatly expanded TRC2 collection such that $>248,000$ unique shRNAs from the TRC collection can potentially be assayed in parallel. To achieve this extraordinary density, GMAP features were synthesized at a $5 \mu \mathrm{m}$ scale, resulting in a 4.84-fold smaller surface area than the $11 \mu \mathrm{m}$ features on the TRCBC array, a commercially unavailable microarray that was previously generated to perform pooled shRNA screens on a subset of the TRC1 shRNA collection [14]. We based the design of the GMAP shRNA features on observations from the TRCBC microarray which included three different, but highly overlapping sequences to detect each shRNA[14]. One of these three feature designs was superior to the others in tests using engineered pools with known relative shRNA compositions (Additional file 1, Figure S1). The GMAP chip starts with the best performing feature design and adds one base from the 21-base shRNA stem sequence to extend the feature length from 21 to 22 bases. Three identical replicates per shRNA barcode feature were included on the GMAP microarray. This strategy was used to maximize signal-to-noise and to identify and potentially correct for any subtle shRNA processing inconsistencies without significantly impacting specificity.

In order to achieve consistent results, a simple-to-follow standard operating procedure (SOP) was developed for preparing samples for hybridization (see Additional file 2 for detailed protocols and recipes). As part of the 
Table 1 Description of the features on the UT GMAP 1.0

\begin{tabular}{llllll}
\hline Probe ID & Description & Unique probes & Replicates & Total number of probes & Probe length \\
\hline hORF & Human ORFeome & 134901 & 1 & 134901 & 25 \\
huORF & HuGene ORFs & 58087 & 1 & 58087 & 25 \\
HP & shRNA sequences (mouse and human) & 248049 & 3 & 744147 & 22 \\
HPC & shRNA negative controls & 138 & 33 & 4554 & 22 \\
HSPI & Hybridization spike-in probes & 200 & 25 & 5000 & 22 \\
HPTMM & shRNA mismatch control probes & 8097 & 3 & 24291 & 22 \\
TAG & Yeast barcode probes & 26801 & 3 & 80403 & 20 \\
yORF & Yeast open reading frames & 11421 & 1 & 11421 & 25 \\
Total & NA & $\mathbf{4 8 7 6 9 4}$ & NA & $\mathbf{1 0 6 2 8 0 4}$ & NA \\
\hline
\end{tabular}

SOP optimization process, a standardized amount of shRNA probe was hybridized to the GMAP array. Hybridizations were performed with quantities of $1 \mu \mathrm{g}, 2 \mu \mathrm{g}$, and $3 \mu \mathrm{g}$ of purified shRNA probe (see Materials and Methods) that was generated from a plasmid pool of 78,432 (78 k) human shRNAs (Additional file 1, Figure S2a). $2 \mu$ g of probe gave the best signal-to-noise ratio and was therefore chosen as the standard quantity for all $78 \mathrm{k}$ pool hybridizations. For pools of different complexity, the amount of probe applied to GMAP is altered proportionally compared to the $2 \mu \mathrm{g}$ used for $78 \mathrm{k}$ pools (for example $\sim 1.35 \mu \mathrm{g}$ for $54 \mathrm{k}$ pools). In addition to optimizing the quantity of probe, a range of hybridization and washing temperatures were tested using $2 \mu \mathrm{g}$ of $78 \mathrm{k}$ shRNA pool probe. Hybridization was tested at $40^{\circ} \mathrm{C}$ and $45^{\circ} \mathrm{C}$, with washing tested at $30^{\circ} \mathrm{C}$ and $35^{\circ} \mathrm{C}$. Hybridization at $40^{\circ} \mathrm{C}$, with array washing at $30^{\circ} \mathrm{C}$ was found to provide the highest probe signal with minimal signal from features corresponding to shRNAs not included in the pool (Additional file 1, Figure S2b).

To assess GMAP chip performance with complex shRNA populations, probe generated from the $78 \mathrm{k}$ human shRNA pool or a different $78 \mathrm{k}$ mouse shRNA pool was hybridized and analyzed. Signal intensity histograms and cumulative distribution plots generated from the collected data indicate that the signal intensities for probes corresponding to shRNAs present in the $78 \mathrm{k}$ pools were well resolved from the background signal of GMAP features corresponding to shRNAs not present in the pools (Additional file 1, Figure S3a,b). The small overlap between the signals for features matched to either of the human or mouse shRNA pools versus the features that have no matching shRNA in the corresponding pools indicates low rates of library composition errors and cross-hybridization. A surface plot of intensities for all shRNA features on the GMAP array for the human $78 \mathrm{k}$ pool versus the mouse $78 \mathrm{k}$ pool indicates that large pools of equal complexity can be easily resolved from each other (Spearman correlation $\mathrm{R}$ $<0.01$ ) and from shRNA features not corresponding to either experimental pool (Figure 1a).
One major application of pooled shRNA screens is to perform negative selection screens, so called "dropout screens", in cancer cell lines to identify cancer-essential genes that represent potential therapeutic targets. To assess the performance of the GMAP in deconvolving genome-scale shRNA drop-out screens, we first calculated a minimum signal in the reference data for inclusion in analysis. This was accomplished by adding 1.96 standard deviations (the $95 \%$ confidence limit) to the mean background log signal measured on human $78 \mathrm{k}$ pool hybridizations, yielding a threshold of $\log _{2}=7.89$ (Additional file 1, Figure S3c). Consequently, 89.9\% of the shRNA signals in the reference data exceeded this threshold and were retained for analysis. We simulated shRNA dropout screens by altering the concentration of sub-fractions of the human $78 \mathrm{k}$ pool in two different shRNA dilution experiments. The first approach utilized four different $78 \mathrm{k}$ human pools, each containing the same 78,432 shRNAs. Approximately 70,100 shRNAs were present in equal concentration in all four pools, while an identical sub-fraction of $\sim 8,300$ shRNAs were either undiluted (the reference "Even pool"), or diluted 4-fold, 16-fold, or 64-fold (the " $4 x$ ", "16x" and " $64 x$ " pools" respectively). Distribution plots of shRNA $\log _{2}$ signal intensity difference between the diluted pools and the Even pool demonstrate that the diluted subpools can be distinctly resolved from each other (Figure 1b). In a second approach, we constructed a single $78 \mathrm{k}$ shRNA pool (the " $2 \mathrm{x}-20 \mathrm{x}$ " pool) in which, relative to the bulk population, four distinct subsets of $\sim 8400$ shRNAs each were diluted 2-fold, 5-fold, 10-fold and 20-fold, respectively. Similar to the previous experiment, distribution plots of shRNA signal difference between diluted pools and the reference population in the Even pool demonstrate that the diluted subpools are resolvable (Figure 1c). We did observe that the signal reduction in diluted subpools on the GMAP chip is not directly proportional to the dilution factor, indicating that changes in microarray signal are not linearly proportional to shRNA concentration across the full observed signal range. In particular, the subpool dilution signals are 


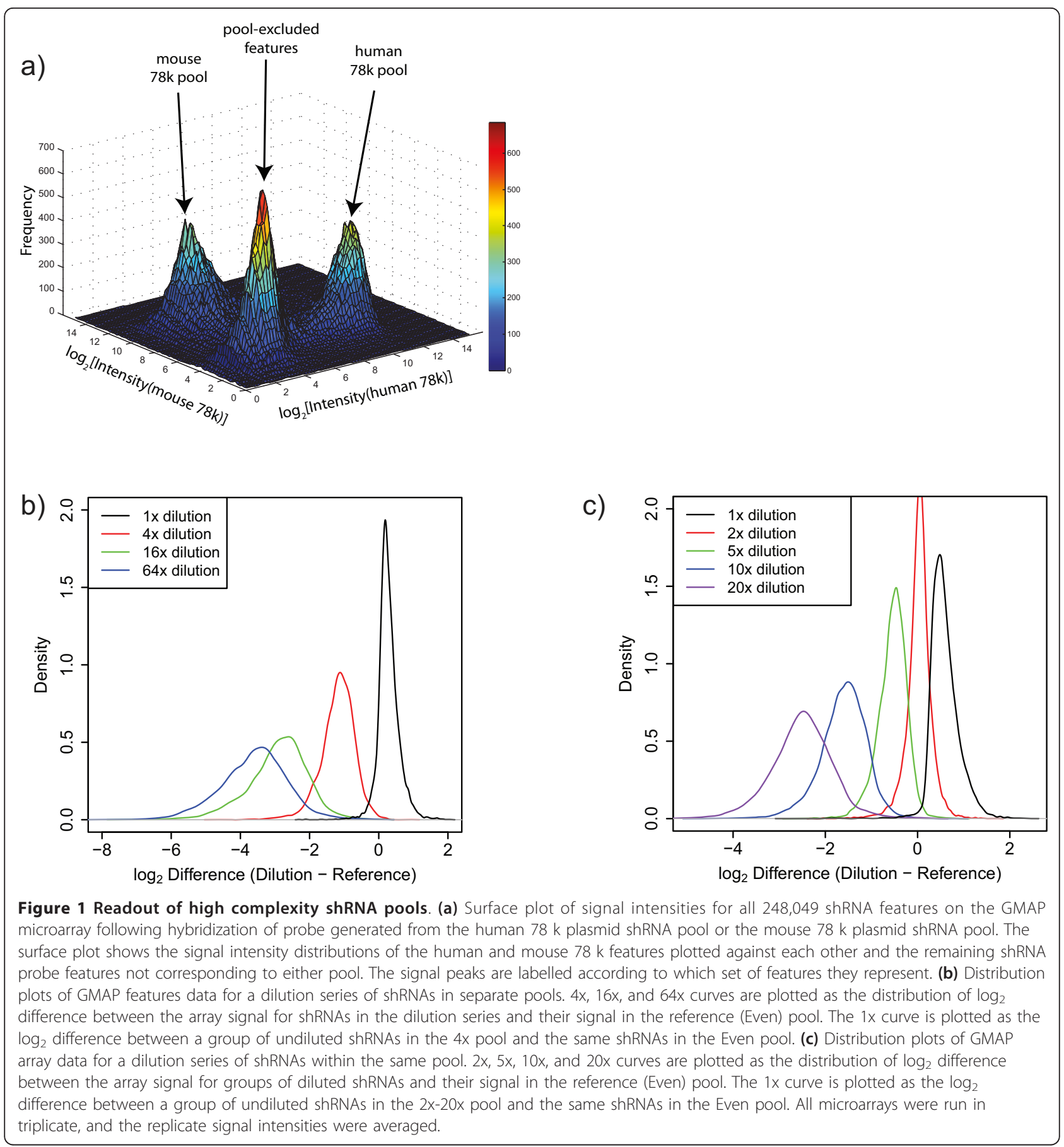

spaced more closely, or compressed, than would be expected for a linear concentration versus signal relationship.

To assess the performance of GMAP relative to the lower density and previous generation TRCBC array, we amplified shRNA probes from genomic DNA extracted from human BT-474 cells infected with a $\sim 54,000$ shRNA pool of lentiviruses corresponding to all available human shRNA features on the TRCBC chip, divided the probe and hybridized samples to both TRCBC and GMAP chips. Comparison of signal intensity between the two different array formats revealed a Spearman correlation coefficient of $\mathrm{R}=0.96$ (Additional file 1, Figure S4). This result confirms that moving from $11 \mu \mathrm{m}$ feature sizes to $5 \mu \mathrm{m}$ feature sizes does not cause any significant reduction in signal strength or specificity, agreeing with reported observations on yeast barcode arrays [26]. 
To compare the BAR-seq strategy [20] with microarray detection for shRNA barcodes, we used Illumina next-generation sequencing technology to enumerate barcodes in the same five human shRNA $78 \mathrm{k}$ pools described above (Even, 4x, 16x, 64x and 2x-20x). Sequencing libraries were prepared from these pools via essentially the same approach used for generating labelled shRNA barcodes for the GMAP array, followed by an additional amplification step to incorporate adapter regions (see Materials and Methods). Between 22.3 million and 25.6 million mapped sequence reads for each of the five shRNA plasmid pools were obtained, yielding a median number of reads per shRNA per $78 \mathrm{k}$ pool of between 107 and 162 (Table 2). Notably, 73,073 (93.4\%) of expected shRNA sequences were detected in the combined data from all five shRNA pools sequenced (>121 million mapped reads) and 68,420 (87.5\%) of expected shRNA barcodes were detected in the human $78 \mathrm{k}$ Even pool alone (Table 2). Comparison of the sequence read frequency to GMAP intensity signal for shRNA barcodes in the Even pool resulted in a positive Spearman correlation of $\mathrm{R}=0.37$ (Figure 2a). By omitting shRNA clones that were not enumerated in the sequencing data by at least 16 reads and with a signal intensity of at least $\log _{2}=7.89$ on the GMAP array, the correlation improved to 0.42 . While both the sequencing and the chip hybridization methods provide an assessment of shRNA relative concentration that is reproducible, the modest level of correlation between the signals for these two types or readouts indicates that they have significantly different relationships to absolute shRNA concentration.

A major concern in using large shRNA pools with microarray detection strategies is the extent to which cross-hybridization occurs. To address this issue, we measured the frequency of significant signals from GMAP shRNA features that did not correspond to

Table 2 Summary of shRNA pool deconvolution by nextgeneration sequencing

\begin{tabular}{llllll}
\hline Statistic & Even & $\mathbf{4 x}$ & $\mathbf{1 6 x}$ & $\mathbf{6 4 x}$ & $\mathbf{2 - 2 0 x}$ \\
\hline $\begin{array}{l}\text { Reads passed } \\
\text { filter }\end{array}$ & 22407757 & 24596943 & 24793754 & 24304519 & 25684516 \\
$\begin{array}{l}\text { Mapped reads } \\
\text { Unmapped }\end{array}$ & 22296083 & 24504006 & 24687271 & 24235485 & 25605983 \\
reads & 111674 & 94937 & 106483 & 69034 & 78533 \\
$\begin{array}{l}\text { Unmapped } \\
\text { reads \% }\end{array}$ & 0.5 & 0.39 & 0.43 & 0.28 & 0.31 \\
$1^{\text {st }}$ quartile & 40 & 34 & 26 & 35 & 21 \\
$\begin{array}{l}\text { Median } \\
\text { Mean }\end{array}$ & 153 & 149 & 140 & 162 & 107 \\
$3^{\text {rd }}$ quartile & 315 & 352.4 & 351.6 & 350.7 & 370.2 \\
Max & 17960 & 19360 & 21750 & 20000 & 29460 \\
Uncounted & 9784 & 10748 & 10045 & 10983 & 10739 \\
\hline & & & & & 385 \\
\hline
\end{tabular}

constituents of a pool of shRNA probes. Specifically, we examined features corresponding to the $78 \mathrm{k}$ mouse pool for signal when the $78 \mathrm{k}$ human pool was hybridized. Features on GMAP that had 100\% identity to shRNAs in both the mouse and human pools were removed from the analysis. After doing so, we found that only 2637 of 77,690 (3.39\%) mouse $78 \mathrm{k}$ pool features had significant signal $\left(\log _{2} \geq 7.89\right)$. From this finding, we infer that amongst the human shRNA features, the cross-hybridization rate from human $78 \mathrm{k}$ pooled probe would be similar.

Frequency distribution plots of sequencing read counts for the Even, 4x, 16x, 64x and 2-20x pools (Figure $2 \mathrm{~b}, \mathrm{c}$ ) show similar characteristics to the distributions generated for the same pools by microarray detection on the GMAP (Figure 1b,c) with some exceptions. First, the distributions tended to be slightly wider for sequence data. Second, the distributions for sequence data exhibit a linear relationship that more accurately reflects the actual experimental dilution of shRNAs in the dilution pools. In other words, the distribution curves for sequencing data tend to center over the correct folddilution. These observations demonstrate that sequencing barcode pools results in linear, quantifiable signals whereas microarray detection displays nonlinear signal, a behaviour previously observed by Pierce et al. [27]. In addition, the dynamic range of signal obtained from GMAP is compressed relative to deep sequencing (Additional file 1, Figure S5). However, these differences aside, overall, GMAP detection of shRNA sequences was similar to sequencing-based deconvolution in its sensitivity and quantitative reproducibility over a range of shRNA concentrations.

Microarray experiments have historically suffered from subtle to substantial differences in data produced from the same or similar templates when performed on different dates, by different users, or in different locations [28]. A candidate method to enable detection and/or correction of these differences is to include a standard set of synthetic oligonucleotide probes that are "spiked" into array hybridization mixtures in defined amounts. These hairpin spike-in (HPSI) probes, designed to have identical length and similar sequence characteristics to shRNAs, may be used as yardsticks for artifact detection and data normalization methods. We replicated 25 clusters of 200 HSPI features across the GMAP (Table 1). Spatially localized signal intensity fluctuations between HSPI clusters may indicate potentially poor hybridization or washing performance, contamination, or physical damage to the array surface. As a trial, 12 HSPI probes were tested in replicate array hybridizations to examine their hybridization characteristics and it was found that they behaved in a dose-dependent manner similar to labelled shRNA probes (Additional file 1, Figure S6). 
a)

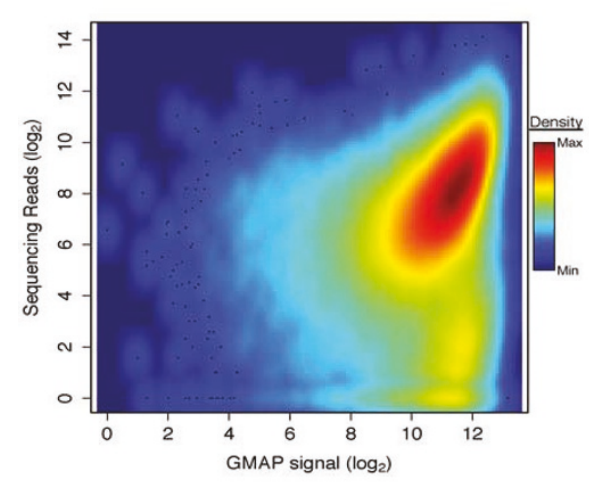

c)

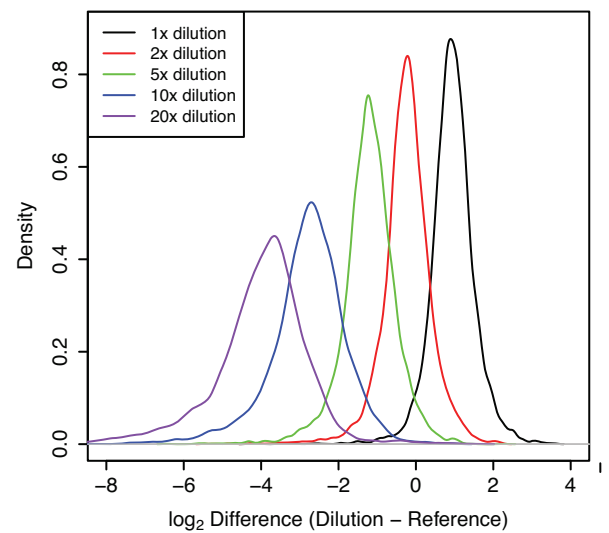

e)

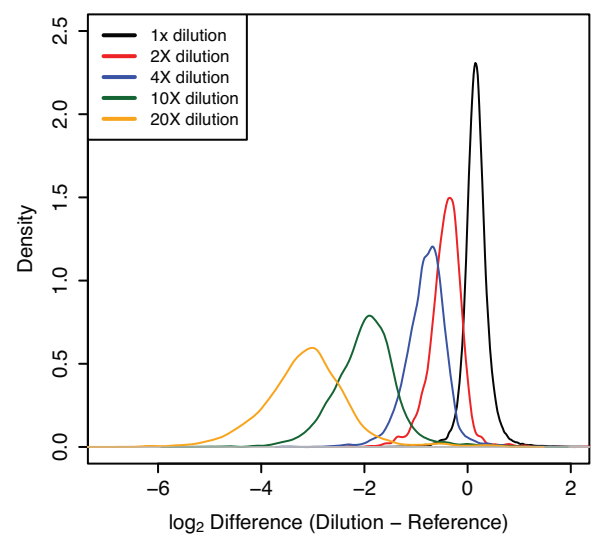

b)

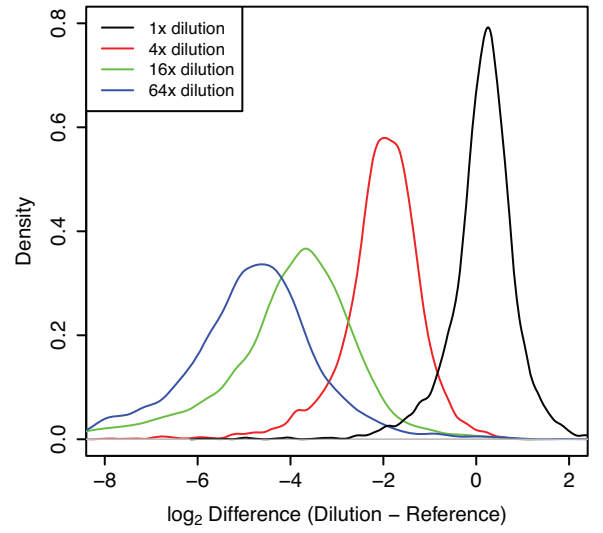

d)

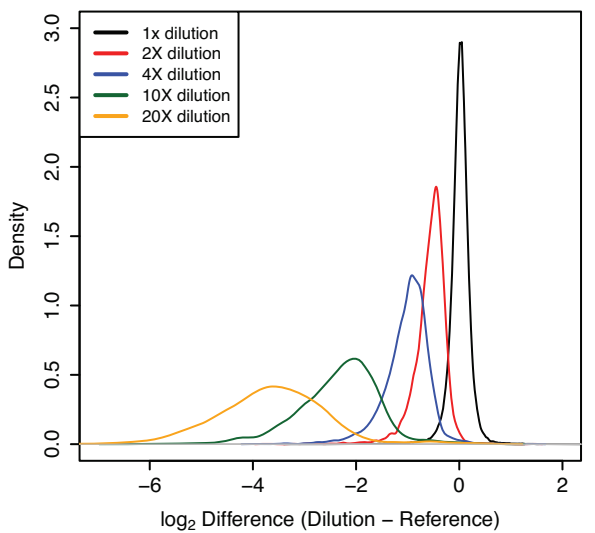

Figure 2 Comparison of sequencing and GMAP performance with high complexity shRNA pools. (a) Scatter plot of GMAP array signal intensity (X-axis) versus sequencing read number (Y-axis) for shRNA clones from the human Even shRNA pool. (b) Distribution plots of Illumina sequencing data for a dilution series of shRNAs in separate pools. $4 x, 16 x$, and $64 x$ curves are plotted as the distribution of $\log _{2}$ difference between the number of sequencing reads for shRNAs in the dilution series and their read count in the reference (Even) pool. The $1 x$ curve is plotted as the $\log _{2}$ difference between a group of undiluted shRNAs in the $4 x$ pool and the same shRNAs in the Even pool. (c) Distribution plots of Illumina sequencing data for a dilution series of shRNAs within the same pool. 2x, 5x, 10x, and 20x curves are plotted as the distribution of $\log _{2}$ difference between the number of sequencing reads for groups of diluted shRNAs and their read count in the reference (Even) pool. The $1 x$ curve is plotted as the $\log _{2}$ difference between a group of undiluted shRNAs in the $2 x-20 x$ pool and the same shRNAs in the Even pool. (d) Distribution plots of GMAP features data for a dilution series of shRNAs contained within sub fractions of a 90,000 shRNA pool where the probe was amplified from shRNA plasmid template DNA. (e) Distribution plots of GMAP features data for a dilution series of shRNAs contained within sub fractions of a 290,000 shRNA pool where the probe was amplified from genomic DNA of A549 cells infected with lentiviral pools. 2x, $5 x, 10 x$, and 20x curves are plotted as the distribution of $\log _{2}$ difference between the array signal for groups of diluted shRNAs in the $90 \mathrm{k}$ Dilution pool and their signal in the $90 \mathrm{k}$ Reference pool. The $1 \times$ curve is plotted as the $\log _{2}$ difference between a group of undiluted shRNAs in the $90 \mathrm{k}$ Dilution pool and the same shRNAs in the $90 \mathrm{k}$ Reference pool. 
To examine GMAP performance in a simulated cell screening experiment, two large pools of 90,408 shRNAs, each targeting $\sim 18,000$ human genes were constructed. For the "90 k Reference" pool, all of the shRNA plasmids were combined at approximately equal concentrations, while a dilution series pool, the "90 k Dilution", contained four sub-sets of $\sim 3,500$ shRNAs each that were diluted 2-fold, 4-fold, 10-fold or 20-fold with respect to their counterparts in the $90 \mathrm{k}$ Reference pool. Distribution plots of shRNA $\log _{2}$ signal difference between the $90 \mathrm{k}$ Dilution and the $90 \mathrm{k}$ Reference pools demonstrate that the diluted sub fractions are clearly resolvable (Figure $2 \mathrm{~d}$ ). We generated lentivirus from the $90 \mathrm{k}$ Reference and $90 \mathrm{k}$ Dilution pools that was subsequently used to infect A549 cells. Genomic DNA prepared from these cells containing integrated shRNAexpressing cassettes was used as template for probe generation and GMAP hybridization. The resulting distribution plots of $\log _{2}$ signal difference between the $90 \mathrm{k}$ Dilution and $90 \mathrm{k}$ Reference pools post-infection (Figure 2e) were very similar to those achieved with probe generated from plasmid template for the $90 \mathrm{k}$ pools and 78 $\mathrm{k}$ pools. A scatter plot comparing data for the $90 \mathrm{k}$ Reference pool derived from plasmid and genomic DNA template reveals excellent correlation $(R>0.97$, see Additional file 1, Figure S7) further demonstrating that reliable results can be obtained from pooled cell-based screening experiments with shRNA pools spanning essentially the entire human genome with coverage of 45 shRNAs per gene.

To enable pooled ORF over-expression screening using the GMAP array to detect and quantify ORF sequences, we designed features against 22,449 distinct human ORF sequences in the Mammalian Genome Collection (MGC) (Table 1 and [29,30]). Between 1 and 8 probes were designed against each ORF, with a median of 7 probes per sequence. For comparative purposes, we also included up to three additional features found on the human expression profiling Affymetrix Gene 1.0 ST array for 18,088 of these sequences. To assess the GMAP performance with human ORF hybridization, we developed 41 plasmid pools of entry clones $(15,347$ open reading frames) from the human ORFeome v5.1 collection that were combined to generate a single master pool. Subsequently, 15,347 ORFs were amplified in pooled format with common flanking primers, labelled and hybridized to both the Human Gene 1.0 ST array and GMAP arrays in duplicate (see Materials and Methods). Signal for features shared between the two arrays was highly correlated (Pearson's correlation coefficient $R=0.953$, Figure 3a), with similar distribution of signal across the features for each array (Figure $3 \mathrm{~b}$ ) and similar signal-to-noise ratios (data not shown). These results demonstrate that the GMAP has robust reporting of ORF data, and suggests that the GMAP can be used for a number of human gene assays including human ORF/cDNA overexpression screens.

To compare the dynamic range of signal for huORF and huGene features on the GMAP, five different quantities of probe generated from ORFeome plasmid pools were hybridized to GMAP in duplicate. The resulting data indicated that two-fold changes in probe input produced highly correlated signals across a 16 -fold dynamic range (Figure 3c), thus we combined huORF and huGene features into one feature set. Our methodology to detect ORF sequences on microarrays depends on common flanking primers that amplify entire open reading frames. This provides an opportunity to examine the utility of the three sets of ORF features on the GMAP microarray, including the 22-mer hairpin features designed to detect shRNA barcodes. The signal concordance between sequence identity-conserved hORF features and shRNA features on GMAP was interchangeable across the entire spectrum of probe concentrations (Figure $3 \mathrm{~d}$ ). This concordance can be further exploited to expand the feature set for each gene to obtain more accurate measurements of ORFs in a given hybridization mix, which is particularly important for discriminating isoforms in a polyclonal library of open reading frames.

GMAP also contains triplicate copies of features for the collection of $\sim 16,00020$ nucleotide Yeast Knockout Collection barcodes[2], and single copies of $\sim 12,00025$ nucleotide yeast ORF-specific features (Table 1). The yeast ORFs represented on this array are identical to those found on the Tag4 barcode array with two distinct probes designed against each of 5718 yeast ORF sequences. These features can serve as additional controls as they are non-homologous to the shRNA or human ORFeome probes on the GMAP. The yeast features on the GMAP display comparable performance to the TAG4 microarray (Affymetrix) from which they are derived (data not shown).

A major hurdle to using a new microarray platform is the informatics associated with data extraction and analysis. The GMAP chip contains several subsets of features, some of which can be organized as traditional Affymetrix feature sets as well as features for interrogation of short input DNA sequences (shRNA barcodes). Extraction of signal from these arrays can be done with the Affymetrix Power Tool (APT) collection. To help aid adoption of the GMAP, we have developed a Java application to allow data reorganization, graphical summaries and downstream analysis of the datasets extracted from these arrays (files and applications available online at http:// chemogenomics.med.utoronto.ca/supplemental/gmap/). This application makes use of a variety of $\mathrm{R}$ and Bioconductor libraries (see Materials and Methods). The R 

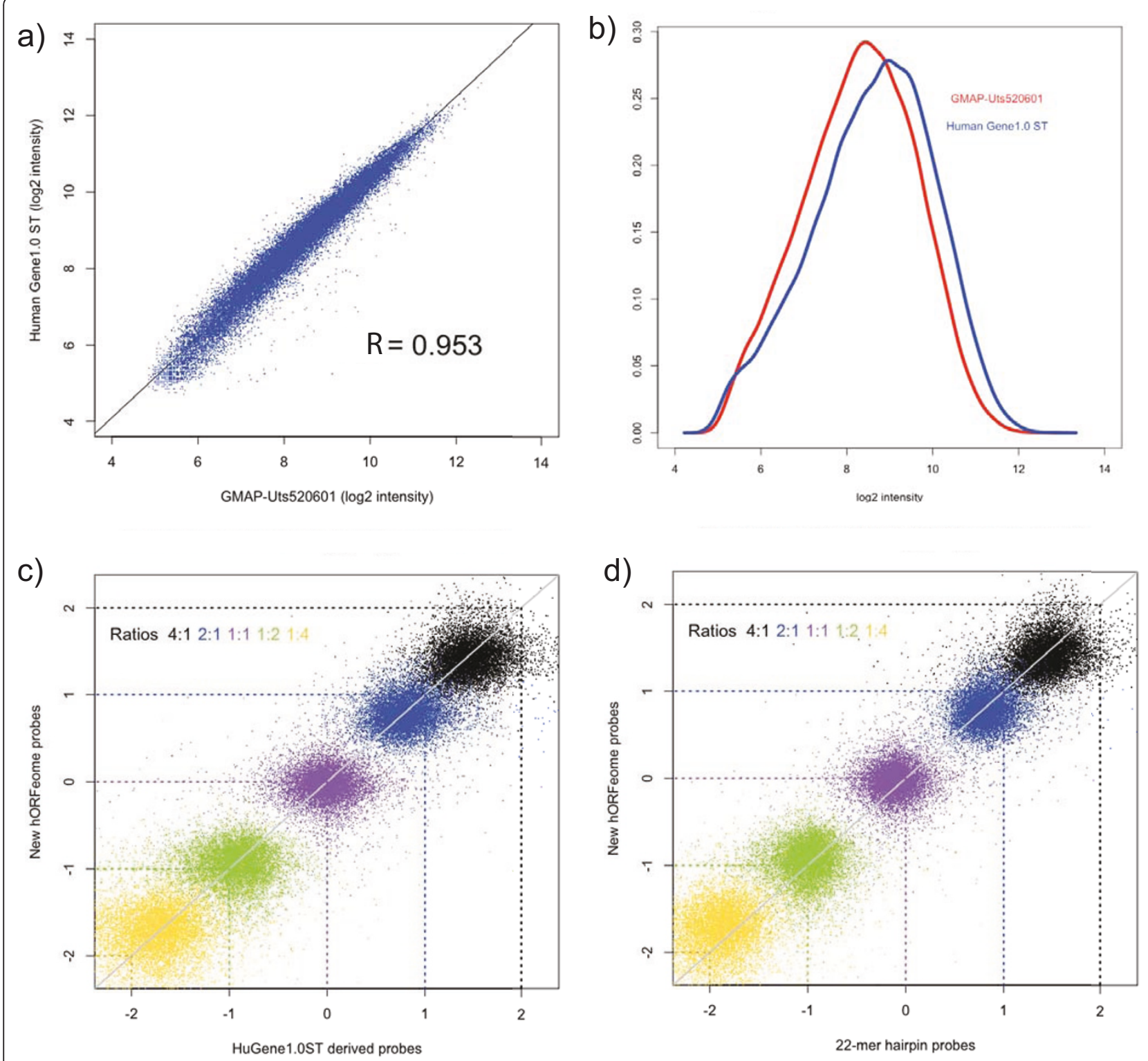

Figure 3 Quality of human ORF features on GMAP chip. (a) Intensity signals from the GMAP array (X-axis) plotted against signals from the Human Gene 1.0 ST array (Y-axis) following amplification of human ORFeome v5.1 pools and hybridization of the resulting probe to each of these arrays. Common features between the two arrays were used to calculate the Pearson correlation coefficient. (b) Distribution of signal intensities from replicate GMAP or Human 1.0 ST arrays as described in (a) for shared features. Hybridization behaviour for the huORF features compared to the (c) huGene features or the (d) 22-mer hairpin features for corresponding genes.

functions that we have developed for this analysis will also be available as scripts/libraries to allow end users to work directly in the $\mathrm{R}$ environment. The current functionality of the application includes methods to extract signals into a single tab-delimited file for subsets of features and GC-matched background signal, normalization procedures, routines to generate signal ratios against one or more reference chips with options to fine-tune analyses and standard or user-defined annotation files for merging analyzed signal.

\section{Conclusions}

Our goal was to develop a comprehensive and standardized microarray platform for quantification of pooled screening results using commercially available gene expression modulation libraries for human, mouse and budding yeast cells. The advantages of a standardized microarray are many-fold. First, a highly validated microarray is invaluable to research labs that want to adopt compatible screening approaches but do not have extensive resources to build, test and optimize a custom 
barcode microarray themselves. The GMAP presents an accessible solution because the obstacle of designing, constructing, and testing of the array as well as development of rigorous SOPs has already been overcome. Second, screening costs are reduced since the GMAP supports multiple commercially available functional screening approaches in a single solution. As such, individual labs or facilities do not have to re-optimize hybridization conditions for different microarray platforms, and a single framework can be used for data extraction and quality control analysis. Third, a standard microarray permits use of uniform methods across the many laboratories using these gene modulation libraries. Lastly, a multipurpose array can serve as a useful validation tool as new technologies for detecting barcodes utilizing deep sequencing mature and become more widely used. To enable facile adoption of the GMAP array for large-scale screen deconvolution, we have also provided a series of supplemental protocols and software tools to help extract data from functional screens using commercially available gene modulation libraries.

A thorough comparison of barcode detection strategies for large, complex shRNA pools has been lacking in the literature. We undertook such a comparison with pools comprised of $\sim 78,000$ and $\sim 90,000$ distinct lentiviral shRNAs, the largest such shRNA screening pools described to date. We directly compared microarray detection with deconvolution by Illumina deep sequencing and conclude that, at least in the current context, data quality is comparable using either method. For many or most users, the array-based read-out is currently more cost-effective on a per-sample basis when costs per sample are tabulated. Equally important as cost for many researchers is the turn-around time required for sample preparation, sample run time and data acquisition/processing, which is currently accomplished more rapidly using microarrays. Sample run time on the GMAP chip spans 18 hours between probe application, hybridization, washing and scanning. Typical microarray platform equipped labs or core facilities should be able to easily accommodate more than 30 experimental samples in a typical work-week.

In addition to examining a plasmid pool containing $>$ 78,000 shRNA clones, we also simulated a cell-based dropout screen with a larger pool of $>90,000$ shRNA clones. Genomic DNA was isolated from each of the transduced populations and shRNA barcodes were detected using the GMAP. Importantly, the resolution of barcode detection was nearly equivalent whether the starting template was from plasmid DNA or genomic DNA. This experiment demonstrates that with the protocols developed here, the GMAP offers an effective and efficient means of screening very large pools in a cellular context. Our trials with complex shRNA and human
ORF pools demonstrate that the GMAP array is capable of efficiently quantifying human and mouse genomescale gene modulation screens. As well, the GMAP chip detection performance is equally suited for quantifying shRNA abundance in lower complexity pools. The GMAP array is not limited to use with the pooled reagents described here - in fact, any screen which uses the yeast deletion barcodes or TRC shRNA sequences as molecular barcodes can be analyzed using the GMAP array. Further generations of GMAP, utilizing even higher feature-count microarrays, could be expanded to include other shRNA and/or ORF libraries. However, benefits of such an expansion would have to be weighed against the increased cost of such expanded arrays. Generating specific arrays corresponding to different shRNA libraries might be more efficient and cost effective. In summary, this cost-effective platform provides any academic laboratory with access to a standardized array and a suite of methods and analytical tools to perform systematic genome-scale genetics on mammalian cells.

\section{Materials and methods}

\section{Cell lines and growth conditions}

BT-474 and A549 cells were obtained from ATCC and maintained in DMEM (Wisent Inc.) + 10\% FBS (Life Technologies-Invitrogen) at $37^{\circ} \mathrm{C}$ and $5 \% \mathrm{CO}_{2}$.

\section{HSPI probe design}

Spike-in sequence-feature combinations were selected so that their perfect match Tm profiles (computed by MELTING[31]) would be similar to shRNA-feature combinations, but any mismatch Tm profiles would be significantly lower than the lowest shRNA-feature Tm profile. We computed Tm only for instances in which spike-in sequences had at least 13 bases of sequence identity to known shRNA features. Sequences could share 13 bases of identity anywhere within the designed 21-mer. Candidate sequences with fewer than 13 bases of identical sequence to any shRNA feature sequence were retained without $\mathrm{Tm}$ evaluation as unlikely to cross-hybridize, and candidate sequences with more than 19 bases of sequence similarity were eliminated outright without evaluating Tm. The top 200 sequences were selected for inclusion as spike-in features on the GMAP array.

\section{Pooled libraries}

All shRNA pools and constructs in this study were derived from the RNAi Consortium lentiviral libraries $[11,14,22]$ available from Sigma-Aldrich and ThermoFisher-Open Biosystems. The human and mouse $78 \mathrm{k}$ shRNA lentivirus plasmid pools were assembled by combining equal proportions of ten sub-pools of $\sim 8,000$ clones each targeting either $\sim 78,000$ human or $\sim 78,000$ 
mouse transcripts. Dilution pools were constructed by the same method, except that diluted sub-pool fractions were reduced in their representation in the $78 \mathrm{k}$ pool by the indicated amounts. Lentivirus pools were generated from pooled lentiviral plasmid DNA. $90 \mathrm{k}$ plasmid pools were constructed by combining 3500-4000 member shRNA sub-pools together. These sub-pools consisted of concentration-normalized plasmid. Dilutions were prepared as described for $78 \mathrm{k}$ pools. The human ORF pool was derived from the human ORFeome v5.1 clones $[23,24]$ available through ThermoFisher-Open Biosystems. 41 subpools of plasmid DNA were generated from 15,347 entry clones where each subpool contained $\sim 380$ human ORFs. A master hORF pool was derived from equivalent amounts of each of the 41 subpools normalized for the number of hORFs in each subpool.

\section{Pooled lentiviral infections}

$7 \times 10^{7}$ A549 cells per replicate were infected with either $90 \mathrm{k}$ Reference or $90 \mathrm{k}$ Dilution lentiviral shRNA pools at an MOI of 0.3-0.4. After four days of selection in puromycin-containing medium to eliminate uninfected cells, genomic DNA was prepared from shRNAcontaining cell populations (Blood Maxi prep kit, Qiagen). $7 \times 10^{7}$ BT474 cells were infected with the human shRNA pool at an MOI of 0.3-04. After two days of selection in puromycin-containing medium to eliminate uninfected cells, genomic DNA was prepared from shRNA-containing cell populations.

\section{shRNA probe preparation (half-hairpin barcodes)}

To create microarray probe from shRNA pools, a master mixture for each sample containing template DNA (78 k shRNA plasmid pools $=250 \mathrm{pg}, 90 \mathrm{k}$ plasmid shRNA pools $=288 \mathrm{pg}$, genomic DNA $=30 \mu \mathrm{g}), 2 \mathrm{x}$ PCR buffer, $2 x$ enhancer solution, $300 \mu \mathrm{M}$ each dNTP, $900 \mathrm{nM}$ each oligonucleotide primer (PCR_B-fw 5'-Biotin-AATGGACTATCATATGCTTACCGTAACTTGAA-3' and PCR_rev 5'-TGTGGATGAATACTGCCATTTGTCTCGAGGTC-3'), 1 mM MgSO 4 , 45 units of Platinum Pfx polymerase (Invitrogen), and water to $1200 \mu \mathrm{l}$ was created and divided into $100 \mu \mathrm{l}$ aliquots. The amplification reaction was performed by denaturing once at $94^{\circ} \mathrm{C}$ for 5 minutes, followed by $\left(94^{\circ} \mathrm{C}\right.$ for 15 seconds, $55^{\circ} \mathrm{C}$ for 15 seconds, $68^{\circ} \mathrm{C}$ for 20 seconds) $\mathrm{x} 30,68^{\circ} \mathrm{C}$ for $5 \mathrm{~min}$ utes, then cooling to $4^{\circ} \mathrm{C}$.

When electrophoresed on a $2 \%$ agarose gel, it is expected that there should be a preponderance of an apparent 178bp PCR product, and as little as possible of an apparent $225 \mathrm{bp}$ product. Both bands on the gel represent amplified shRNA barcode sequences, but the upper, slower migrating band is composed of two DNA strands in a cruciform structure, centered around the palindromic shRNA sequence, which is not suitable for further processing into a GMAP probe. DNA in this structure is resistant to restriction enzyme activity at the XhoI restriction site between the shRNA palindrome sequences. Application of PCR product containing a mixture of two apparently different products to a denaturing urea gel resolves a single size species of DNA (data not shown). If PCR reactions are favouring cruciform product, reducing the number of cycles in the PCR reaction will favour the linear product (data not shown). PCR products are immediately purified using the QIAquick PCR purification kit (Qiagen) since long-term storage at $4^{\circ} \mathrm{C}$ or $-20^{\circ} \mathrm{C}$ enables the conversion of linear product to cruciform DNA. The purified 178bp PCR product is then digested with XhoI (New England Biolabs) for 2 hours at $37^{\circ} \mathrm{C}$ to generate a thermo-stable half-hairpin probe. The digested samples are separated on $2 \%$ agarose gels with lanes large enough to accommodate $150 \mu \mathrm{l}$ of sample. Using sterile materials, the half-hairpin probes ( 106bp) are excised from the gel and purified using a gel extraction kit (QIAGEN). To remove remaining salts, the probe DNA may be passed through a PCR cleanup column, and eluted in a final volume of $30 \mu \mathrm{l}$.

\section{GMAP shRNA probe hybridization}

Microarrays were pre-hybridized by one wash with $40^{\circ} \mathrm{C}$ $10 \mathrm{mM} \mathrm{NaOH}$, followed by incubation at $40^{\circ} \mathrm{C}$ for 10 minutes with rotation at $40 \mathrm{rpm}$ with a second volume of $10 \mathrm{mM} \mathrm{NaOH}$. The arrays were then slowly washed with 3-5 $\mathrm{ml} 6 x$ SSPE, $0.0001 \%$ Tween 20 , followed by filling and incubation for 10 minutes at $40^{\circ} \mathrm{C}$ with rotation at $40 \mathrm{rpm}$ with $0.0005 \%$ Triton X-100. Arrays were then emptied, rinsed twice, then filled with $6 \mathrm{x}$ SSPE, $0.0001 \%$ Tween 20 , and incubated for 2 hours at $40^{\circ} \mathrm{C}$ with rotation at $40 \mathrm{rpm}$.

Hybridization solutions consisted of $2 \mu \mathrm{g}$ of probe for $78 \mathrm{k}$ shRNA pools $(2.3 \mu \mathrm{g}$ for $90 \mathrm{k}$ shRNA pools $)$ in buffer containing $1 \mathrm{x}$ MES, $0.89 \mathrm{M} \mathrm{NaCl}, 20 \mathrm{mM}$ EDTA, $0.0001 \%$ Tween $20,0.5 \mathrm{mg} / \mathrm{ml} \mathrm{BSA}, 0.1 \mathrm{mg} / \mathrm{ml}$ herring sperm DNA, $0.05 \mathrm{nM}$ biotinylated B2 oligo (Affymetrix), 10\% DMSO, $20 \mu \mathrm{M}$ each blocking oligos (Block_1 5'AATGGACTATCATATGCTTACCGTAACTTGAA-3', Block_2 5'-TTCAAGTTACGGTAAGCATATGATAGTCCATT-3', Block_3 5'-GTATTTCGATTTCTTGGCTTTATATATCTTGTGGAAAGGACGAAACACCG3', Block_4 5'-CGGTGTTTCGTCCTTTCCACAAGATATATAAAGCCAAGAAATCGAAATAC-3'), and sterile water to a final volume of $138 \mu \mathrm{l}$. Samples in buffer were denatured at $95^{\circ} \mathrm{C}$ for 10 minutes, incubated at $40^{\circ}$ $\mathrm{C}$ for 5 minutes, collected by centrifugation then applied to arrays, which were incubated for 16 hours at $40^{\circ} \mathrm{C}$ at $60 \mathrm{rpm}$. Arrays were stained with SAPE labeling mix (1x MES staining buffer, $2 \mathrm{mg} / \mathrm{ml}$ BSA, $10 \mu \mathrm{g} / \mathrm{ml}$ streptavidin-phycoerythrin), and washed on an Affymetrix fluidics station, then scanned. More complete shRNA probe 
preparation and hybridization protocols are included in Additional file 2.

\section{Illumina sequencing}

PCR product was prepared as described above for GMAP shRNA probe preparation except that 27 cycles of PCR were employed when amplifying from pooled plasmid DNA. Purified PCR product DNA was used as a template for a second round of amplification in order to incorporate Illumina adapter sequences. Each $100 \mu \mathrm{l}$ reaction contained $5 \mathrm{ng}$ of template, $2 \mathrm{x}$ PCR buffer, $2 \mathrm{x}$ enhancer solution, $300 \mu \mathrm{M}$ each dNTP, 900nM each for Adapter A (5'-AATGATACGGCGACCACCGAAATGGACTATCATATGCTTACCGTAACTTGAA-3') and Adapter B (5'-CAAGCAGAAGACGGCATACGATGTGGATGAATACTGCCATTTGTCTCGAGGTC-3'), $1 \mathrm{mM} \mathrm{MgSO}_{4}, 3.75$ units of Platinum Pfx polymerase, and water to $100 \mu \mathrm{l}$. The PCR reaction was performed by denaturing at $94^{\circ} \mathrm{C}$ for 5 minutes, followed by $\left(94^{\circ} \mathrm{C}\right.$ for 15 seconds, $55^{\circ} \mathrm{C}$ for 15 seconds, $68^{\circ} \mathrm{C}$ for $20 \mathrm{sec}$ onds) $\mathrm{x} 16,68^{\circ} \mathrm{C}$ for 5 minutes, then cooling to $4^{\circ} \mathrm{C}$. The resulting $218 \mathrm{bp}$ product was purified by electrophoresis in $2 \%$ agarose followed by gel extraction. Libraries were initially quantified by the Quant-IT fluorescent assay (Invitrogen), and fragment size was confirmed using a Bioanalyzer high sensitivity chip in a 2100 Bioanalyzer (Agilent Technologies). Libraries were diluted to $\sim 15 \mathrm{nM}$ concentration, and concentration was confirmed using commercially available qPCR standard on 1:1000 dilutions of library samples. DNA templates were then diluted to $8 \mathrm{pM}$ as per the Illumina cBot user guide, and clusters were generated on a single read flowcell using the Illumina cBot. Each dilution pool was run in a separate lane. Using an Illumina GAIIx instrument, sequence was collected for 22 bases using the primer SeqshRNA (5'-GATTTCTTGGCTTTATATATCTTGTGGAAAGGACGAAACACCG-3').

\section{Illumina data processing}

Raw reads were generated using Illumina's Offline Base Caller software (v1.61). Reads that passed quality filtering were extracted from the QSEQ files and merged into FASTQ files using a custom BASH script. Reads were aligned to a synthetic chromosome, constructed by interspersing the hairpin sense strand sequence with 79nt of random sequence, using MAQ v0.7.1 [32] with default parameters. The synthetic chromosome and hairpin index are available upon request. Aligned sequences were matched to individual hairpin IDs if the alignment start site corresponded with the correct starting nucleotide of the hairpin sequence (i.e.: sequences with mismatches on the first nucleotide were removed) and the MAQ quality score was $\geq 50$. This quality threshold allowed one mismatch over the 21 nt sequence. If a read mapped to one of the 227 shRNA barcode sequences duplicated in the shRNA library, it was counted once for each of the duplicate hairpin IDs. Reads were determined in a single replicate experiment, and counts were $\log _{2}$ transformed prior to further analysis.

\section{human ORF probe preparation and hybridization}

Human ORFs were amplified from the master hORF pool where each hORF was contained within the pDONR223 plasmid. Five reactions were carried out in a $50 \mu \mathrm{L}$ volume containing $25 \mu \mathrm{L}$ of $2 \mathrm{x}$ Phusion Flash High-Fidelity Master Mix (Finnzymes), 400nM of each primer and $10 \mathrm{ng}$ of plasmid. The reaction times and temperature were 1 minute at $98^{\circ} \mathrm{C}$ for 1 cycle; $10 \mathrm{sec}$ onds at $98^{\circ} \mathrm{C}, 20$ seconds at $60^{\circ} \mathrm{C}$, and 4 minutes at $72^{\circ} \mathrm{C}$ for 30 cycles; 10 minutes at $72^{\circ} \mathrm{C}$ for 1 cycle. The sequences of the forward and reverse primers specific for the cloned human ORFs were 5'-CACGACGTTGTAAAACGACGGCCAGTC-3' and 5'-GAGCTGCCAGGAAACAGCTATGACCATG-3' respectively. PCR products were purified (Qiagen) and pooled. Purified PCR product was biotinylated (8 reactions, 500ng per reaction) using a BioPrime DNA labelling kit (Invitrogen) and unincorporated biotin-14-dCTP was removed by passing the samples through Sephadex G-50 columns (GE Healthcare). Microarrays were pre-hybridized by adding $130 \mu \mathrm{L}$ of Hybridization buffer and incubating for 10 minutes at $45^{\circ} \mathrm{C}$ and $60 \mathrm{rpm}$. The hybridization mix contained $2 \mathrm{x}$ hybridization buffer, $5 \mathrm{nM}$ B2 oligo, 50x Denhardt's solution and biotinylated PCR product. Different amounts of labelled probe were added to Affymetrix arrays and hybridized at $45^{\circ} \mathrm{C}$ for 17 hours with a rotation of $60 \mathrm{rpm}$. GMAP-UTS520601 Affymetrix arrays were hybridized with $7 \mu \mathrm{g}, 3.5 \mu \mathrm{g}, 1.75 \mu \mathrm{g}, 0.875$ $\mu \mathrm{g}$ or $0.4375 \mu \mathrm{g}$ of biotinylated PCR product obtained from the human ORFeome 5.1 collection. The Human Gene 1.0 ST arrays were hybridized with $3.5 \mu \mathrm{g}$ of sample. Probe was hybridized to the arrays for 17 hours at $45 \mathrm{C}$ and $60 \mathrm{rpm}$. Arrays were stained with SAPE labelling mix (2x MES staining buffer, $20 \mathrm{mg} / \mathrm{mL}$ BSA, and 1 $\mathrm{mg} / \mathrm{mL}$ streptavidin-phycoerythrin), washed on an Affymetrix fluidics station and scanned.

\section{GMAP data extraction and processing}

Feature signal was extracted from the GMAP and TRCBC arrays using Affymetrix Power Tools v.1.12.0 (APT, http://www.affymetrix.com). The GMAP chip contains triplicate features per shRNA, which we summarized by using the median value. GC-background (GCbg) correction for non-specific probe binding was performed with APT, using feature signals derived from 33,894 GC-background probes on the array. Normalization of replicate arrays was performed with the Bioconductor affy package (v1.26.1) in R, using Cyclic Loess 
based on the 'MA-plot' of pairs of arrays. Distribution plots generated from the raw, GCbg-corrected, and Loess-normalized signal intensities demonstrated that GCbg-correction served to increase the differentiation between signal from the features corresponding to shRNA pool probes and those features on the GMAP without corresponding partners in the probe pool. while normalization reduced the variance between replicates (Additional file 1, Figure S8).

\section{Web-based tool for GMAP}

We have developed a Java front-end through which this extraction and analysis can be accomplished by users outside our laboratories. The current functionality of the application includes the following:

1. Extraction of signal from a set of Affymetrix. CEL files into a single tab-delimited file. The extracted signal can be limited to a specific subset of features and GC-matched background signal can be subtracted.

2. Signal across chips can be normalized using a variety of procedures. Graphical output is generated for assessment of normalization.

3. Currently, the primary use of this chip is for assessment of strain abundance during pooled growth in a variety of systems that correspond to features on this array. This includes strains with integrated molecular barcodes, transduced with shRNA sequences, or hORF sequences. The Java application includes tools to calculate signal ratios against one or more reference chips with a variety of options available to fine-tune the analysis.

4. Extracted feature signal datasets can be further reduced by limiting to features relevant to specific pools. Standard or user-defined annotation files can be merged with the analyzed signal.

This application, $\mathrm{R}$ scripts, links to supporting software and examples of use are available on our supplementary website http://chemogenomics.med.utoronto. $\mathrm{ca} /$ supplemental/gmap/.

\section{Additional material}

Additional file 1: contains additional figures S1-S8 and the corresponding figure legends.

Additional file 2: contains details methods for GMAP probe generation and hybridization as well as recipes and reagents.

List of Abbreviations

APT: Affymetrix Power Tool; DNA: Deoxyribonucleic Acid; GMAP: Gene Modulation Array Platform; HPSI: Hairpin Spike-In; huORF: Human ORF; MGC:
Mammalian Genome Collection; ORF: Open Reading Frame; shRNA: Short hairpin RNA; SOP: Standard Operating Procedure; TRC: The RNAi Consortium; TRCBC: The RNAi Consortium Barcode; RNA: Ribonucleic Acid

\section{Acknowledgements}

This work was supported by the Canadian Foundation for Innovation (C.N. and J.M.), the Ontario Institute for Cancer Research (J.M.), and the Canadian Institutes for Health Research (C.N., G.G. and J.M.) and by the Canadian Cancer Society (Research Grant \#020380).

We thank members of The RNAi Consortium (TRC2) for helpful discussions and support. The authors thank Thomas Green, Tashfeen Bhimdi and Matthew Greene for assistance in the selection and design of hairpin spikein probes (HSPI).

\section{Author details}

'Donnelly Centre and Banting \& Best Department of Medical Research, University of Toronto, Toronto, Canada. ${ }^{2}$ Department of Molecular Genetics, University of Toronto, Toronto, Canada. ${ }^{3}$ Broad Institute, Cambridge, USA. ${ }^{4}$ Department of Pharmaceutical Sciences, University of Toronto, Toronto, Canada.

\section{Authors' contributions}

All authors read and approved the final manuscript. TK developed GMAP shRNA protocols (based on earlier work from EE and KCO), and performed shRNA probe amplification. LEH (along with AS, GSC, SG, RA, TK CN, JM, DER) was responsible for GMAP design. LEH and AS developed Java-based GMAP data extraction tools. LEH assisted with data extraction in shRNA sequencing experiments. DK prepared shRNA probe samples and performed GMAP shRNA pool hybridizations. KRB and JLYK performed provided statistical analysis of shRNA GMAP and sequencing data. RA, AMS, AA performed ORF over-expression experiments and analysis on GMAP. KB and DK provided shRNA reagents. TD performed shRNA sequencing reactions. GSC and XY prepared TRC shRNAs, $90 \mathrm{k}$ shRNA pools and $90 \mathrm{k}$ shRNA infected cell genomic DNA. Experimental work was carried out in the labs of GG, DER, JM and CN. TK and JM wrote the bulk of the manuscript, with contributions from all other authors. JM and CN directed the project.

Received: 4 November 2010 Accepted: 6 May 2011

Published: 6 May 2011

\section{References}

1. Giaever G, Chu AM, Ni L, Connelly C, Riles L, Veronneau S, Dow S, LucauDanila A, Anderson K, Andre B, Arkin AP, Astromoff A, El-Bakkoury M, Bangham R, Benito R, Brachat S, Campanaro S, Curtiss M, Davis K, Deutschbauer A, Entian KD, Flaherty P, Foury F, Garfinkel DJ, Gerstein M, Gotte D, Guldener U, Hegemann JH, Hempel S, Herman Z, et al: Functional profiling of the Saccharomyces cerevisiae genome. Nature 2002, 418:387-391.

2. Winzeler EA, Shoemaker DD, Astromoff A, Liang H, Anderson K, Andre B, Bangham R, Benito R, Boeke JD, Bussey H, Chu AM, Connelly C, Davis K, Dietrich F, Dow SW, El Bakkoury M, Foury F, Friend SH, Gentalen $E_{t}$ Giaever G, Hegemann JH, Jones T, Laub M, Liao H, Liebundguth N, Lockhart DJ, Lucau-Danila A, Lussier M, M'Rabet N, Menard P, et al: Functional characterization of the $S$. cerevisiae genome by gene deletion and parallel analysis. Science 1999, 285:901-906.

3. Costanzo M, Baryshnikova A, Bellay J, Kim Y, Spear ED, Sevier CS, Ding H, Koh JL, Toufighi K, Mostafavi S, Prinz J, St Onge RP, VanderSluis B, Makhnevych T, Vizeacoumar FJ, Alizadeh S, Bahr S, Brost RL, Chen Y, Cokol M, Deshpande R, Li Z, Lin ZY, Liang W, Marback M, Paw J, San Luis BJ, Shuteriqi E, Tong AH, van Dyk N, et al: The genetic landscape of a cell. Science 327:425-431.

4. Giaever G, Shoemaker DD, Jones TW, Liang H, Winzeler EA, Astromoff A, Davis RW: Genomic profiling of drug sensitivities via induced haploinsufficiency. Nat Genet 1999, 21:278-283.

5. Hillenmeyer ME, Fung E, Wildenhain J, Pierce SE, Hoon S, Lee W, Proctor M, St Onge RP, Tyers M, Koller D, Altman RB, Davis RW, Nislow C, Giaever G: The chemical genomic portrait of yeast: uncovering a phenotype for all genes. Science 2008, 320:362-365.

6. Tong AH, Evangelista $M$, Parsons $A B, X u H$, Bader GD, Page N, Robinson M, Raghibizadeh S, Hogue CW, Bussey H, Andrews B, Tyers M, Boone C: 
Systematic genetic analysis with ordered arrays of yeast deletion mutants. Science 2001, 294:2364-2368.

7. Tong $A H$, Lesage $G$, Bader GD, Ding $H, X u H$, Xin X, Young J, Berriz GF, Brost RL, Chang M, Chen Y, Cheng X, Chua G, Friesen H, Goldberg DS, Haynes J, Humphries C, He G, Hussein S, Ke L, Krogan N, Li Z, Levinson JN, Lu H, Menard P, Munyana C, Parsons AB, Ryan O, Tonikian R, Roberts T, et al: Global mapping of the yeast genetic interaction network. Science 2004, 303:808-813.

8. Bernards R, Brummelkamp TR, Beijersbergen RL: shRNA libraries and their use in cancer genetics. Nat Methods 2006, 3:701-706.

9. Chang K, Elledge SJ, Hannon GJ: Lessons from Nature: microRNA-based shRNA libraries. Nat Methods 2006, 3:707-714.

10. Moffat J, Sabatini DM: Building mammalian signalling pathways with RNAi screens. Nat Rev Mol Cell Biol 2006, 7:177-187.

11. Root DE, Hacohen N, Hahn WC, Lander ES, Sabatini DM: Genome-scale loss-of-function screening with a lentiviral RNAi library. Nat Methods 2006, 3:715-719.

12. Berns K, Hijmans EM, Mullenders J, Brummelkamp TR, Velds A, Heimerikx M, Kerkhoven RM, Madiredjo M, Nijkamp W, Weigelt B, Agami R, Ge W, Cavet G, Linsley PS, Beijersbergen RL, Bernards R: A large-scale RNAi screen in human cells identifies new components of the p53 pathway. Nature 2004, 428:431-437.

13. Paddison PJ, Silva JM, Conklin DS, Schlabach M, Li M, Aruleba S, Balija V, O'Shaughnessy A, Gnoj L, Scobie K, Chang K, Westbrook T, Cleary M, Sachidanandam R, McCombie WR, Elledge SJ, Hannon GJ: A resource for large-scale RNA-interference-based screens in mammals. Nature 2004, 428:427-431

14. Luo B, Cheung HW, Subramanian A, Sharifnia T, Okamoto M, Yang X, Hinkle G, Boehm JS, Beroukhim R, Weir BA, Mermel C, Barbie DA, Awad T, Zhou X, Nguyen T, Piqani B, Li C, Golub TR, Meyerson M, Hacohen N, Hahn WC, Lander ES, Sabatini DM, Root DE: Highly parallel identification of essential genes in cancer cells. Proc Natl Acad Sci USA 2008, 105:20380-20385.

15. Luo J, Emanuele MJ, Li D, Creighton CJ, Schlabach MR, Westbrook TF, Wong KK, Elledge SJ: A genome-wide RNAi screen identifies multiple synthetic lethal interactions with the Ras oncogene. Cell 2009, 137:835-848

16. Schlabach MR, Luo J, Solimini NL, Hu G, Xu Q, Li MZ, Zhao Z, Smogorzewska A, Sowa ME, Ang XL, Westbrook TF, Liang AC, Chang K, Hackett JA, Harper JW, Hannon GJ, Elledge SJ: Cancer proliferation gene discovery through functional genomics. Science 2008, 319:620-624.

17. Silva JM, Marran K, Parker JS, Silva J, Golding M, Schlabach MR, Elledge SJ, Hannon GJ, Chang K: Profiling essential genes in human mammary cells by multiplex RNAi screening. Science 2008, 319:617-620.

18. Ngo VN, Davis RE, Lamy L, Yu X, Zhao H, Lenz G, Lam LT, Dave S, Yang L, Powell J, Staudt LM: A loss-of-function RNA interference screen for molecular targets in cancer. Nature 2006, 441:106-110.

19. Pierce SE, Davis RW, Nislow C, Giaever G: Genome-wide analysis of barcoded Saccharomyces cerevisiae gene-deletion mutants in pooled cultures. Nat Protoc 2007, 2:2958-2974.

20. Smith AM, Heisler LE, Mellor J, Kaper F, Thompson MJ, Chee M, Roth FP, Giaever G, Nislow C: Quantitative phenotyping via deep barcode sequencing. Genome Res 2009, 19:1836-1842.

21. Smith AM, Heisler LE, St Onge RP, Farias-Hesson E, Wallace IM, Bodeau J, Harris AN, Perry KM, Giaever G, Pourmand N, Nislow C: Highly-multiplexed barcode sequencing: an efficient method for parallel analysis of pooled samples. Nucleic Acids Res.

22. Moffat J, Grueneberg DA, Yang X, Kim SY, Kloepfer AM, Hinkle G, Piqani B, Eisenhaure TM, Luo B, Grenier JK, Carpenter AE, Foo SY, Stewart SA, Stockwell BR, Hacohen N, Hahn WC, Lander ES, Sabatini DM, Root DE: A lentiviral RNAi library for human and mouse genes applied to an arrayed viral high-content screen. Cell 2006, 124:1283-1298.

23. Lamesch P, Li N, Milstein S, Fan C, Hao T, Szabo G, Hu Z, Venkatesan K, Bethel G, Martin P, Rogers J, Lawlor S, McLaren S, Dricot A, Borick H, Cusick ME, Vandenhaute J, Dunham I, Hill DE, Vidal M: hORFeome v3.1: a resource of human open reading frames representing over 10,000 human genes. Genomics 2007, 89:307-315.

24. Rual JF, Hirozane-Kishikawa T, Hao T, Bertin N, Li S, Dricot A, Li N, Rosenberg J, Lamesch P, Vidalain PO, Clingingsmith TR, Hartley JL, Esposito D, Cheo D, Moore T, Simmons B, Sequerra R, Bosak S, DoucetteStamm L, Le Peuch C, Vandenhaute J, Cusick ME, Albala JS, Hill DE, Vidal M:
Human ORFeome version 1.1: a platform for reverse proteomics. Genome Res 2004, 14:2128-2135.

25. Hoon S, Smith AM, Wallace IM, Suresh S, Miranda M, Fung E, Proctor M, Shokat KM, Zhang C, Davis RW, Giaever G, St Onge RP, Nislow C: An integrated platform of genomic assays reveals small-molecule bioactivities. Nat Chem Biol 2008, 4:498-506.

26. Ammar R, Smith AM, Heisler LE, Giaever G, Nislow C: A comparative analysis of DNA barcode microarray feature size. BMC Genomics 2009, 10:471.

27. Pierce SE, Fung EL, Jaramillo DF, Chu AM, Davis RW, Nislow C, Giaever G: A unique and universal molecular barcode array. Nat Methods 2006, 3:601-603

28. Park PJ, Cao YA, Lee SY, Kim JW, Chang MS, Hart R, Choi S: Current issues for DNA microarrays: platform comparison, double linear amplification, and universal RNA reference. J Biotechnol 2004, 112:225-245

29. Temple G, Gerhard DS, Rasooly R, Feingold EA, Good PJ, Robinson C, Mandich A, Derge JG, Lewis J, Shoaf D, Collins FS, Jang W, Wagner L, Shenmen CM, Misquitta L, Schaefer CF, Buetow KH, Bonner TI, Yankie L, Ward M, Phan L, Astashyn A, Brown G, Farrell C, Hart J, Landrum M, Maidak BL, Murphy M, Murphy T, Rajput B, et al: The completion of the Mammalian Gene Collection (MGC). Genome Res 2009, 19:2324-2333.

30. Rouillard JM, Zuker M, Gulari E: OligoArray 2.0: design of oligonucleotide probes for DNA microarrays using a thermodynamic approach. Nucleic Acids Res 2003, 31:3057-3062.

31. Le Novere N: MELTING, computing the melting temperature of nucleic acid duplex. Bioinformatics 2001, 17:1226-1227.

32. Li H, Ruan J, Durbin R: Mapping short DNA sequencing reads and calling variants using mapping quality scores. Genome Res 2008, 18:1851-1858.

doi:10.1186/1471-2164-12-213

Cite this article as: Ketela et al:: A comprehensive platform for highly multiplexed mammalian functional genetic screens. BMC Genomics 2011 $12: 213$

\section{Submit your next manuscript to BioMed Central and take full advantage of:}

- Convenient online submission

- Thorough peer review

- No space constraints or color figure charges

- Immediate publication on acceptance

- Inclusion in PubMed, CAS, Scopus and Google Scholar

- Research which is freely available for redistribution 